\title{
Ensayo sobre estrategias para documentar el conocimiento en una organización
}

\author{
Isabel Medrano Corrales \\ Licenciada en Geografia e Historia. Archivera \\ Luis Suárez Samaniego \\ Licenciado en Informática. Analista funcional
}

\section{Resumen}

Los sistemas para la gestión del conocimiento, en su vertiente de gestión de la información, enfatizan el valor del conocimiento tácito existente en una organización, considerando su potencial ventaja si llega a transformarse en conocimiento explícito y, por tanto, con posibilidad de ser compartido por todos los miembros. Son objeto de análisis y reflexión en este artículo los procedimientos para materializar la experiencia y sabiduría adquirida por las personas a través de la generación de documentos con distintos grados de automatización. En este sentido, se propone el desarrollo de un repertorio en el que estén representados los objetos más relevantes relacionados con el conocimiento clave para la organización, y a partir de aquí la configuración de un editor de documentos de conocimiento que se publicarán en la intranet corporativa y a los que se podrá acceder a través de distintas formas de búsqueda.

\section{Palabras clave}

Gestión del conocimiento/ Capital intelectual/ Gestión de la información/ Sistema de información/ Intranet corporativa

\section{Introducción}

Este artículo pretende abordar la casuística de un modelo de organización que ya cuenta con un sistema de información con altos requerimientos y una incipiente cultura corporativa, pero que aún está en camino hacia la consecución de una efectiva gestión del conocimiento. Partiendo de esta realidad, se realiza una propuesta para articular mecanismos que faciliten a los miembros de la organización el documentar sus actividades, contemplando aspectos interpretativos y de valoración de los hechos, que quedan fuera de un estricto sistema de automatización del trabajo y de los procedimientos. Y todo esto, teniendo en cuenta la integración con el sistema de información ya existente. Se trataría de poner a disposición de los usuarios una herramienta capaz de editar el texto libre que el redactor quiera dejar reflejado y todos las posibles referencias que quiera incorporar como información de contexto y explicativa de los propios hechos. De esta forma, se les facilitaría a los autores de los documentos el relato y la reconstrucción de la actividad de la que se pretende dejar constancia. El producto resultante estaría incorporado en la intranet corporativa como una plataforma para el acceso al conocimiento de las prácticas de la organización.

\section{Premisas estratégicas y tecnológicas de la organización}

Vamos a desarrollar nuestra hipótesis de trabajo sobre un modelo de organización genérico, que debe cumplir con una serie de condiciones en su sistema de trabajo y de información, sobre el que vamos a proponer la adopción de medidas encaminadas a documentar el conocimiento que las personas que la componen poseen y que no es compartido por el común. Estas condiciones a las que nos referimos son las siguientes:

- Las actividades de la organización han de realizarse conforme a procedimientos normalizados, que estén regulados por escrito, bien sea conformando un Manual de Calidad, o con normas de procedimiento. Por tanto, los procesos administrativos y de negocio han debido ser analizados, redactados y son conocidos por los miembros de la organización, que en el ejercicio de su actividad comprueban la viabilidad de los mismos y proponen posibles modificaciones o mejoras.

- La gestión de la organización debe estar soportada por un Sistema de Información que integre datos y procesos y los haga visible a través de una tecnología tipo intranet.

- Debe reconocerse como línea estratégica de la organización la cultura corporativa, impulsada desde la dirección e interiorizada por los miembros. La percepción por parte de todos de que el fomento del conocimiento compartido es una ventaja competitiva para la organización es un elemento clave a la hora de emprender cualquier actuación encaminada a gestionar el conocimiento.

En el marco del programa para la gestión del conocimiento, vamos a centrarnos en su vertiente de ges- 
tión de la información, y más concretamente en el diseño de una metodología para documentar el conocimiento. En el proceso distinguimos las siguientes fases:

\begin{tabular}{ll}
\hline FASE & PRODUCTO \\
\hline $\begin{array}{l}\text { Identificación del } \\
\text { conocimiento clave }\end{array}$ & $\begin{array}{l}\text { Documento programático de } \\
\text { gestión del conocimiento }\end{array}$ \\
\hline $\begin{array}{l}\text { Selección de las fuentes } \\
\text { de información }\end{array}$ & $\begin{array}{l}\text { Elaboración de un repositorio } \\
\text { de objetos }\end{array}$ \\
\hline $\begin{array}{l}\text { Implantación de la solución } \\
\text { tecnológica }\end{array}$ & Desarrollo de la herramienta \\
\hline $\begin{array}{l}\text { Exteriorización del } \\
\text { conocimiento }\end{array}$ & $\begin{array}{l}\text { Creación y clasificación de } \\
\text { documentos }\end{array}$ \\
\hline $\begin{array}{l}\text { Interiorización del } \\
\text { conocimiento }\end{array}$ & Acceso a los documentos \\
\hline
\end{tabular}

\section{Identificación del conocimiento clave}

Al abordar el programa de gestión del conocimiento, la organización debe, en primera instancia, realizar una labor de análisis para la detección e identificación del conocimiento clave, que son los puntos fuertes de su actividad, en los que radica su particularidad y valor. Y la gestión que sobre ese conocimiento se emprenda, estará destinada a la resolución de los problemas, a la asistencia en la toma de decisiones, a la creación de nuevos productos y servicios, para, en definitiva, propiciar la adaptación y desarrollo de la organización acorde con las nuevas realidades y cambios en el entorno.

Es quizás éste el momento más adecuado para que se produzca en paralelo la oportuna reflexión interna respecto a los posibles cambios en la estructura organizativa. Habida cuenta de la vorágine que habitualmente rige el día a día de las organizaciones con alta carga tecnológica, parece necesaria la incorporación a tiempo parcial de algunos trabajadores a las nuevas tareas de documentar el conocimiento. Disponer, por el contrario, de personal exclusivamente para estas tareas no se considera idóneo, en tanto que puede derivar en un distanciamiento de la realidad de la actividad diaria. Por otro lado, confiar en que el personal va a emplear tiempo para estas nuevas tareas como añadido a su actividad ordinaria es cuando menos arriesgado, a pesar de que existan mecanismos que incentiven estas prácticas.

\section{Selección de las fuentes de información}

A continuación se trataría de localizar en el sistema de información todos los recursos existentes que estén relacionados con el mapa de contenidos definidos como conocimiento clave. Estas fuentes de información pasarían a estar representadas en un repositorio de objetos, entendiendo como objeto cualquier entidad sobre la que almacenar información, sea una norma, una persona (trabajador, cliente), una actividad, etc.

De forma genérica, clasificamos las fuentes de información en las siguientes:

TIPOLOGÍA DE LAS FUENTES DE INFORMACIÓN

Sistemas transaccionales

- Gestión de adquisiciones

- Gestión de ventas

- Gestión de proyectos

- Contabilidad

- Nóminas

Sistemas de información estructurada

- Registro general de documento

- Registro de currícula

- Herramientas colaborativas (agendas, contactos,...)

- Bases de datos especializadas: prensa, legislación

- Programa de gestión de la biblioteca

- Programa de gestión del archivo

Sistemas de información no estructurada

- Enlaces a recursos de internet

- Comunidades virtuales

- Informes externos (estudios de mercado, análisis de la competencia,...)

Herramientas de generación de informes

- Las propias de los sistemas de información existentes

- Data mining

- Dataware house

- Inventario y catálogo del archivo

- Catálogo de la biblioteca

Cabe destacar, en este primer nivel de concepción de los objetos sobre el que generar conocimiento, algunos aspectos que van a venir a modelarlos desde distintas perspectivas y los enriquecerán facilitando su uso y diversificando las formas de explotación:

Versiones. Es conveniente, en función de los requerimientos, que cuando documentemos una experiencia podamos decidir si la referencia que citamos de un objeto es independiente del factor tiempo. Así, a una referencia a un proveedor siempre le pediremos que nos lleve a su teléfono, e-mail, etc. actual, mientras que cuando documentemos la problemática que tuvimos con ese proveedor respecto de un pedido, quizás sea mas interesante que el documento refleje el estado del pedido en el momento anterior a la resolución del problema, ya que posiblemente contenga información relevante. Este mantenimiento de versiones puede realizarse de dos formas bien diferenciadas: generando en el sistema de información subyacente una versión protegida para no ser modificada o copiando los atributos más relevantes del objeto en cuestión para incorporarlos al documento de conocimiento que estamos redactando.

Vistas. Cuando hacemos referencia a un objeto de nuestro repositorio, ison relevantes todos sus atribu- 
tos?, la respuesta en los casos de objetos complejos parece ser claramente no. No es lo mismo la referencia que podamos hacer a un documento, que la cambiante realidad de, por ejemplo, la cuenta de resultados de un proyecto o la situación de tesorería de determinada cuenta bancaria. Parece evidente que no sería óptimo producir una herramienta de gestión de conocimiento que soporte una réplica del propio sistema de información. Por lo tanto, se propone como solución la referencia a vistas parciales de estos objetos, lo cual permitirá mayor agilidad en su ulterior explotación.

Roles. Este aspecto está muy en relación con el anterior, siendo la definición del producto cartesiano de ambos el que en buena medida nos va a facilitar la construcción de un sistema de gestión de conocimiento de eficiente explotación. Entendemos por rol el papel o papeles que, definidos por la organización, juegan cada uno de sus miembros. Así, se puede originar un mismo documento de conocimiento que, según quién lo consulte, se mostrará de una forma u otra para adecuarse a la realidad más cercana al quehacer diario de cada miembro de la organización. Una correcta asociación entre roles y vistas (recordemos: conjunto de atributos de un objeto de la organización) producirán una potente herramienta de gestión de conocimiento.

\section{Implantación de la solución tecnológica}

Recapitulando, hemos hablado de la creación de un repositorio en el que están representados los objetos más relevantes relacionados con el conocimiento clave para la organización, con diversas formas de visualizarlos (vistas), en diferentes momentos (versiones) y para distintas personas (roles). A partir de aquí, proponemos la documentación del conocimiento mediante un editor en el que, además de redactarse con texto libre los hechos que se quieren constatar, permita incluir referencias a los objetos del sistema de información subyacente, diferenciando vistas, versiones y roles. Estas referencias se incorporarían, bien de manera incrustada o pegada en el documento, o bien como un hiperenlace desde el que podemos acceder al objeto referenciado.

A continuación haremos una sucinta referencia a los entornos tecnológicos actualmente disponibles para construir la herramienta de gestión de conocimiento. Si el sistema de gestión empresarial consta de una herramienta propia, el éxito en la construcción del sistema dependerá en buena medida de las facilidades que dé dicha herramienta a adaptarse a otros sistemas. En caso de partir de cero, la solución pasa en buena medida por adoptar una de las tecnologías de gestión de objetos distribuidos, llámese COM o CORBA, que permiten acceder a los objetos de la organización siempre que éstos hayan sido creados (objeto proxy) en el repositorio de objetos del gestor (broker) del sistema. La perfecta integración de esta tecnología con servidores de intranet (IIS, Apache), servidores transaccionales (ITS, MTS), objetos distribuidos (SOAP), gestores de colas asíncronas, etc. conforman el marco ideal para crear nuestro repositorio de objetos empresariales, sus vistas, roles, versiones, etc. junto con el potencial que entraña en cuanto a incorporación de sistemas de gestión documental, multimedia, cursos on-line, páginas web de la intranet que describen la estructura de la organización, webflow para gestión de incidentes, etc. Una vez definido este soporte base, es necesario dotarse de una aplicación para construir el documento sobre conocimiento.

\section{La exteriorización ${ }^{\mid}$del conocimiento}

Una forma común de materializar y transmitir el conocimiento en una organización es a través de los documentos. Los sistemas de calidad hacen hincapié en la exigencia de crear registros de documentos como testimonio de las actividades. Y, en general, los procedimientos de trabajo incorporan requisitos para documentar cada proceso. Pero esta práctica con frecuencia no siempre se cumple por las dificultades de emplear tiempo en documentar una actividad ya realizada, puesto que hay otras tareas prioritarias. Y, sobre todo, estos documentos no suelen aportar información interpretativa sobre la gestión de las actividades; se convierten en registros de datos escuetos donde el autor no llega a abordar un trabajo de redacción comprensiva de los pasos que ha seguido en la consecución de un fin, las referencias internas o externas que ha consultado y de los problemas que ha encontrado para resolverlos.

Estas circunstancias nos llevan a pensar que una de las claves está en la posibilidad de documentar el conocimiento sobre una experiencia concreta simultáneamente a la realización de la misma -la redacción a posteriori siempre estará sujeta a que otra actividad más urgente supedite su realización definitiva-. Para esto, la persona que genere un documento para el conocimiento debe encontrarse en un espacio en el que le resulte rápido trabajar, pueda navegar desde allí a todas las fuentes de información que necesite consultar y tenga la posibilidad de referenciarlas.

Se detectan tres maneras en cuanto a la forma de construir el documento con el editor que proponemos:

De generación manual. Contando con un repositorio de objetos y con la pertinente herramienta de redacción que nos permita acceder de forma sencilla y clara a según que objetos del sistema, documentar el conocimiento de forma libre requiere simplemente poner a disposición del documentalista una interfase que le permita acceder desde la herramienta de redacción al repositorio de objetos. Para optimizar la accesibilidad posterior al documento es necesario definir los objetos y sus versiones, vistas de los objetos y roles a los que está destinado.

De generación automática. La idea básica de este método tipo 'grabadora' consiste en que la herramienta de redacción vaya recogiendo -a partir de las pantallas que se usan para realizar las tareas- los dis- 
tintos pasos y la información que se va consultando o actualizando con objeto de obtener el documento de forma automática. La ventaja de este método radica principalmente en que el propio contexto de desarrollo de trabajo ahorra al autor del documento definir de forma activa roles, versiones, vistas, etc. Efectivamente, este método presupone un esfuerzo inicial de asociación de aspectos de los objetos a las pantallas de trabajo, así como que las actividades a documentar estén integradas en la intranet de la organización.

Generado en un circuito de workflow. Es éste un caso particular del método automático, en el que las tareas se encuentran ya predefinidas y automatizadas en el sistema de información, siguiendo un orden establecido y que afecta a varias personas. La idea consiste en aprovechar los circuitos de workflow y fomentar la redacción de comentarios que los participantes pueden hacer cuando tienen que ejecutar tareas automatizadas a través de dicha herramienta. Estos textos se referirán a problemas aparecidos en el desarrollo de la tarea, solución que se propone,... De los comentarios que los participantes vayan depositando se podrá obtener información útil para ser evaluada por el responsable del proceso y constituirá una fuente de conocimiento para la organización en general.

Una vez finalizado el documento, ya sea por cualquiera de los tres procedimientos mencionados, es necesario clasificarlo para luego poderlo recuperar en una búsqueda. Independientemente de que reciban un tratamiento posterior, es recomendable que el propio autor comience a asignarle algunas taxonomías relativas a las materias sobre las que versa y a su valoración inicial sobre la utilidad del mismo. No sólo convendría tener documentos sobre cómo se deben hacer bien las cosas, sino también experiencias en falso. La organización debe incentivar la proactividad de sus miembros y es por esto que conviene tener guardadas experiencias de éxito relativo que sirvan como repertorio de acciones a no adoptar o 'malas prácticas' que sirvan de referente por eliminación cuando se pretenda abordar una tarea de una forma distinta a las ya conocidas.

Posteriormente sería interesante que el documento no se publique definitivamente en el sistema y quede accesible al resto de la organización hasta que una persona o un grupo de personas con responsabilidades generales sobre el sistema de gestión del conocimiento den su valoración y culminen la clasificación del mismo.

\section{La interiorización ${ }^{2}$ del conocimiento}

Ya hemos llegado a documentar el conocimiento, hemos definido un nuevo objeto en el sistema -el documento sobre conocimiento- configurado a partir de un texto libre enriquecido con referencias a los objetos de la organización. Gracias a este carácter semiestruturado (texto, objetos) el abanico de posibilidades de recuperación de la información se amplía, ofreciéndose los siguientes métodos de búsqueda:

Búsqueda contextual. Consiste en el acceso automático a las fuentes de conocimiento -tanto documentos como cursos, normativa, etc.- en función de la tarea que estemos realizando en un momento dado. El sistema obtiene del usuario registrado en el sistema su rol y en función de la tarea que esté desempeñando le muestra los documentos pertienentes en base a las vistas asociadas a su rol. De forma más refinada, incluso podría acceder a documentos concretos del objeto en cuestión con el que trabaje.

Búsqueda guiada. Los parámetros de búsqueda son ingresados por el usuario y están orientados al objeto. A los ya mencionados de objeto, valores de atributos, rol, vista, se añaden los propios derivados del documento de conocimiento en sí. Así, puede interesarnos los documentos de un determinado autor, o que hayan sido valorados positivamente por un miembro concreto de la organización, etc.

Búsqueda mediante tecnologías de internet. Es la búsqueda sobre el texto libre del documento mediante métodos and/or 'tradicionales' de un indexador web, por proximidad, mediante ejemplos, etc.; o bien usando herramientas más complejas, como las redes neuronales, el reconocimiento de patrones o la búsqueda semántica.

Acceso por navegación en estructuras fijas. No hay una búsqueda con resultados de los que elegimos documentos que vamos revisando. El acceso se realiza navegando sobre estructuras establecidas en la intranet, como pueden ser el diagrama organizativo, los procedimientos internos, el catálogo de cursos online, etc.

Mapa de relaciones. Esta herramienta se presenta como una opción que aporta nuevas perspectivas en la resolución de problemas, al establecerse un entramado de relaciones entre los conceptos que tienen entre sí influencias. El resultado sería un mapa con la representación bidimensional de todos los conceptos relacionados y sus enlaces correspondientes.

La interiorización del conocimiento producida con el acceso y uso de los documentos por parte del equipo de la organización, generará en las personas nuevo conocimiento tácito, de forma que este proceso se convierte en cíclico. Los propios usuarios harán su valoración de la utilidad y la facilidad de acceso a los documentos de conocimiento, retroalimentando así el propio sistema.
2. La interiorización es un proceso de incorporación de conocimiento explícito en conocimiento tácito, que analiza las experiencias adquiridas en la puesta en práctica de los nuevos conocimientos y que se incorpora en las bases de conocimiento tácito de los miembros de la organización en la forma de modelos mentales compartidos o prácticas de trabajo. (Nonaka y Takeuchi, 1995) 